\title{
Optimal Two Parameter Bounds for the Seiffert Mean
}

\author{
Hui Sun, Ying-Qing Song, and Yu-Ming Chu \\ School of Mathematics and Computation Science, Hunan City University, Yiyang 413000, China \\ Correspondence should be addressed to Yu-Ming Chu; chuyuming2005@yahoo.com.cn \\ Received 30 April 2013; Revised 18 July 2013; Accepted 18 July 2013 \\ Academic Editor: Zhihua Zhang
}

Copyright (C) 2013 Hui Sun et al. This is an open access article distributed under the Creative Commons Attribution License, which permits unrestricted use, distribution, and reproduction in any medium, provided the original work is properly cited.

We obtain sharp bounds for the Seiffert mean in terms of a two parameter family of means. Our results generalize and extend the recent bounds presented in the Journal of Inequalities and Applications (2012) and Abstract and Applied Analysis (2012).

\section{Introduction}

For $a, b>0$ with $a \neq b$, the Seiffert mean $T(a, b)$, root mean square $S(a, b)$, and contraharmonic mean $C(a, b)$ are defined by

$$
\begin{gathered}
T(a, b)=\frac{a-b}{2 \arctan [(a-b) /(a+b)]}, \\
S(a, b)=\sqrt{\frac{a^{2}+b^{2}}{2}}, \\
C(a, b)=\frac{a^{2}+b^{2}}{a+b},
\end{gathered}
$$

respectively. It is well known that the inequalities

$$
T(a, b)<S(a, b)<C(a, b)
$$

hold for all $a, b>0$ with $a \neq b$.

Recently, $T(a, b), S(a, b)$, and $C(a, b)$ have been the subject of intensive research. In particular, many remarkable inequalities and properties for these means can be found in the literature [1-8].
For $\alpha, \beta, \lambda, \mu \in(1 / 2,1)$, very recently Chu et al. $[9,10]$ proved that the inequalities

$$
\begin{aligned}
S(\alpha a & +(1-\alpha) b, \alpha b+(1-\alpha) a) \\
& <T(a, b) \\
& <S(\beta a+(1-\beta) b, \beta b+(1-\beta) a), \\
C(\lambda a+(1-\lambda) b, \lambda b+(1-\lambda) a) & \\
& <T(a, b) \\
& <C(\mu a+(1-\mu) b, \mu b+(1-\mu) a)
\end{aligned}
$$

hold for all $a, b>0$ with $a \neq b$ if and only if $\alpha \leq(1+$ $\left.\sqrt{16 / \pi^{2}-1}\right) / 2, \beta \geq(3+\sqrt{6}) / 6, \lambda \leq(1+\sqrt{4 / \pi-1}) / 2$, and $\mu \geq(3+\sqrt{3}) / 6$.

$$
\text { Let } t \in(1 / 2,1), p \geq 1 / 2 \text {, and }
$$

$$
Q_{t, p}(a, b)=C^{p}(t a+(1-t) b, t b+(1-t) a) A^{1-p}(a, b),
$$

where $A(a, b)=(a+b) / 2$ is the classical arithmetic mean of $a$ and $b$. Then from (2), (3), and (7) we clearly see that

$$
\begin{gathered}
Q_{t, 1 / 2}(a, b)=S(t a+(1-t) b, t b+(1-t) a), \\
Q_{t, 1}(a, b)=C(t a+(1-t) b, t b+(1-t) a),
\end{gathered}
$$

and $Q_{t, p}(a, b)$ is strictly increasing with respect to $t \in(1 / 2,1)$ for fixed $a, b>0$ with $a \neq b$. 
It is natural to ask what are the greatest value $t_{1}=t_{1}(p)$ and the least value $t_{2}=t_{2}(p)$ in $(1 / 2,1)$ such that the double inequality

$$
Q_{t_{1}, p}(a, b)<T(a, b)<Q_{t_{2}, p}(a, b)
$$

holds for all $a, b>0$ with $a \neq b$ and $p \geq 1 / 2$. The aim of this paper is to answer this question; our main result is the following Theorem 1.

Theorem 1. If $t_{1}, t_{2} \in(1 / 2,1)$ and $p \in[1 / 2, \infty)$, then the double inequality

$$
Q_{t_{1}, p}(a, b)<T(a, b)<Q_{t_{2}, p}(a, b)
$$

holds for all $a, b>0$ with $a \neq b$ if and only if $t_{1} \leq 1 / 2+$ $\left[\sqrt{(4 / \pi)^{1 / p}-1}\right] / 2$ and $t_{2} \geq 1 / 2+\sqrt{3 p} /(6 p)$.

Remark 2. If we take $p=1 / 2$ and $p=1$ in Theorem 1 , then inequality (10) reduces to inequalities (5) and (6), respectively.

\section{Proof of Theorem 1}

In order to prove Theorem 1 we need two lemmas, which we present in this section.

Lemma 3 (see [11, Theorem 1.25]). For $-\infty<a<b<\infty$, let $f, g:[a, b] \rightarrow \mathbb{R}$ be continuous on $[a, b]$ and differentiable on $(a, b)$; let $g^{\prime}(x) \neq 0$ on $(a, b)$. If $f^{\prime}(x) / g^{\prime}(x)$ is increasing (decreasing) on $(a, b)$, then so are

$$
\frac{f(x)-f(a)}{g(x)-g(a)}, \quad \frac{f(x)-f(b)}{g(x)-g(b)} .
$$

If $f^{\prime}(x) / g^{\prime}(x)$ is strictly monotone, then the monotonicity in the conclusion is also strict.

Lemma 4. Let $u \in[0,1], p \geq 1 / 2$ and

$$
f_{u, p}(x)=p \log \left(1+u x^{2}\right)-\log x+\log \arctan x .
$$

Then

(1) $f_{u, p}(x)>0$ for $x \in(0,1)$ if and only if $3 p u \geq 1$;

(2) $f_{u, p}(x)<0$ for $x \in(0,1)$ if and only if $1+u \leq(4 / \pi)^{1 / p}$.

Proof. By (12) and simple computations one has

$$
\begin{gathered}
\lim _{x \rightarrow 0} f_{u, p}(x)=0, \\
f_{u, p}(1)=p \log (1+u)+\log \left(\frac{\pi}{4}\right),
\end{gathered}
$$

$$
\begin{aligned}
& f_{u, p}^{\prime}(x) \\
& =\frac{2 p u x}{1+u x^{2}}+\frac{1}{\left(1+x^{2}\right) \arctan x}-\frac{1}{x} \\
& =\left(u\left[(2 p-1) x^{2}\left(1+x^{2}\right) \arctan x+x^{3}\right]\right. \\
& \left.\quad-\left[\left(1+x^{2}\right) \arctan x-x\right]\right) \\
& \quad \times\left(x\left(1+x^{2}\right)\left(1+u x^{2}\right) \arctan x\right)^{-1} \\
& =\frac{(2 p-1) x^{2}\left(1+x^{2}\right) \arctan x+x^{3}}{x\left(1+x^{2}\right)\left(1+u x^{2}\right) \arctan x}[u-g(x)],
\end{aligned}
$$

where

$$
g(x)=\frac{\left(1+x^{2}\right) \arctan x-x}{(2 p-1) x^{2}\left(1+x^{2}\right) \arctan x+x^{3}} .
$$

Let $g_{1}(x)=\arctan x-x /\left(1+x^{2}\right)$ and $g_{2}(x)=(2 p-$ 1) $x^{2} \arctan x+x^{3} /\left(1+x^{2}\right)$. Then $g_{2}(x)$ is strictly increasing in $(0,1)$ :

$$
g(x)=\frac{g_{1}(x)}{g_{2}(x)}, \quad g_{1}(0)=g_{2}(0)=0,
$$

$$
\frac{g_{1}^{\prime}(x)}{g_{2}^{\prime}(x)}=\frac{1}{(2 p-1)\left[\left(1+x^{2}\right)^{2} \arctan x\right] / x+p x^{2}+p+1} \text {. }
$$

It is not difficult to verify that the function $x \rightarrow[(1+$ $\left.\left.x^{2}\right)^{2} \arctan x\right] / x$ is strictly increasing from $(0,1)$ onto $(1, \pi)$; hence (18) implies that $g_{1}^{\prime}(x) / g_{2}^{\prime}(x)$ is strictly decreasing in $(0,1)$. Therefore, $g(x)$ is strictly decreasing in $(0,1)$ follows from Lemma 3 and (17) together with the monotonicity of $g_{2}(x)$ and $g_{1}^{\prime}(x) / g_{2}^{\prime}(x)$. Moreover, making use of l'Hôpital's rule we get

$$
\begin{gathered}
\lim _{x \rightarrow 0} g(x)=\frac{1}{3 p}, \\
g(1)=\frac{\pi-2}{(2 p-1) \pi+2} .
\end{gathered}
$$

We divide the proof into three cases.

Case $1(u \geq 1 /(3 p))$. Then from (15) and (19) together with the monotonicity of $g(x)$ we conclude that $f_{u, p}(x)$ is strictly increasing in $(0,1)$. Therefore $f_{u, p}(x)>0$ for $x \in(0,1)$ follows from (13) and the monotonicity of $f_{u, p}(x)$.

Case $2(u \leq(\pi-2) /[(2 p-1) \pi+2])$. Then from (15) and (20) together with the monotonicity of $g(x)$ we clearly see that $f_{u, p}(x)$ is strictly decreasing in $(0,1)$. Therefore $f_{u, p}(x)<0$ for $x \in(0,1)$ follows from (13) and the monotonicity of $f_{u, p}(x)$.

Case $3((\pi-2) /[(2 p-1) \pi+2]<u<1 /(3 p))$. Then from (15), (19), and (20) together with the monotonicity of $g(x)$ 
we know that there exists $x_{0} \in(0,1)$ such that $f_{u, p}(x)$ is strictly decreasing in $\left(0, x_{0}\right)$ and strictly increasing in $\left(x_{0}, 1\right)$. Therefore, $f_{u, p}(x)<0$ in $(0,1)$ if and only if $f_{u, p}(1) \leq 0$ follows from (13) and the piecewise monotonicity of $f_{u, p}(x)$, which (14) gives immediately $1+u \leq(4 / \pi)^{1 / p}$.

Proof of Theorem 1. Since both $Q_{t, p}(a, b)$ and $T(a, b)$ are symmetric and homogeneous of degree 1 , without loss of generality, we assume that $a>b$. Let $x=(a-b) /(a+b) \epsilon$ $(0,1)$. Then from (1) and (7) we get

$$
\begin{aligned}
& \log \left(\frac{Q_{t, p}(a, b)}{T(a, b)}\right) \\
& =\log \left(\frac{Q_{t, p}(a, b)}{A(a, b)}\right)-\log \left(\frac{T(a, b)}{A(a, b)}\right) \\
& =p \log \left[1+(1-2 t)^{2} x^{2}\right]-\log x+\log \arctan x .
\end{aligned}
$$

Therefore, Theorem 1 follows from Lemma 4 and (21).

\section{Acknowledgments}

This work was supported by the Natural Science Foundation of China (Grant nos. 11071069 and 11171307) and the Natural Science Foundation of Zhejiang Province (Grant no. LY13A010004).

\section{References}

[1] H. J. Seiffert, “Aufgabe $\beta 16$,” Die Wurzel, vol. 29, pp. 221-222, 1995.

[2] P. A. Hästö, "A monotonicity property of ratios of symmetric homogeneous means," Journal of Inequalities in Pure and Applied Mathematics, vol. 3, no. 5, article 71, 23 pages, 2002.

[3] E. Neuman and J. Sándor, "On the Schwab-Borchardt mean," Mathematica Pannonica, vol. 14, no. 2, pp. 253-266, 2003.

[4] E. Neuman and J. Sándor, "On the Schwab-Borchardt meanII," Mathematica Pannonica, vol. 17, no. 1, pp. 49-59, 2006.

[5] H. Liu and X.-M. Ju, "The optimal convex combination bounds for Seiffert's mean," Journal of Inequalities and Applications, vol. 2011, Article ID 686834, 9 pages, 2011.

[6] W.-D. Jiang, "Some sharp inequalities involving reciprocals of the Seiffert and other means," Journal of Mathematical Inequalities, vol. 6, no. 4, pp. 593-599, 2012.

[7] W.-D. Jiang and F. Qi, "Some sharp inequalities involving Seiffert and other means and their concise proofs," Mathematical Inequalities \& Applications, vol. 15, no. 4, pp. 1007-1017, 2012.

[8] J. Sandor, "Trigonometric and hyperbolic inequalities," submitted, http://arxiv.org/abs/1105.0859.

[9] Y.-M. Chu, S.-W. Hou, and Z.-H. Shen, "Sharp bounds for Seiffert mean in terms of root mean square," Journal of Inequalities and Applications, vol. 2012, article 11, 2012.

[10] Y.-M. Chu and S.-W. Hou, "Sharp bounds for Seiffert mean in terms of contraharmonic mean," Abstract and Applied Analysis, vol. 2012, Article ID 425175, 6 pages, 2012.

[11] G. D. Anderson, M. K. Vamanamurthy, and M. K. Vuorinen, Conformal Invariants, Inequalities, and Quasiconformal Maps, John Wiley \& Sons, New York, NY, USA, 1997. 


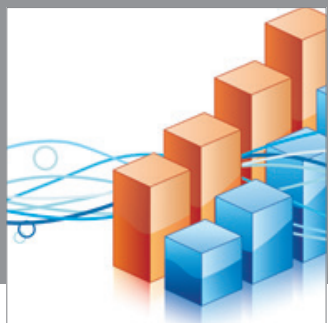

Advances in

Operations Research

mansans

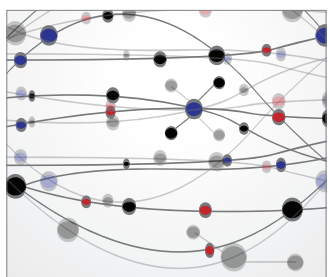

The Scientific World Journal
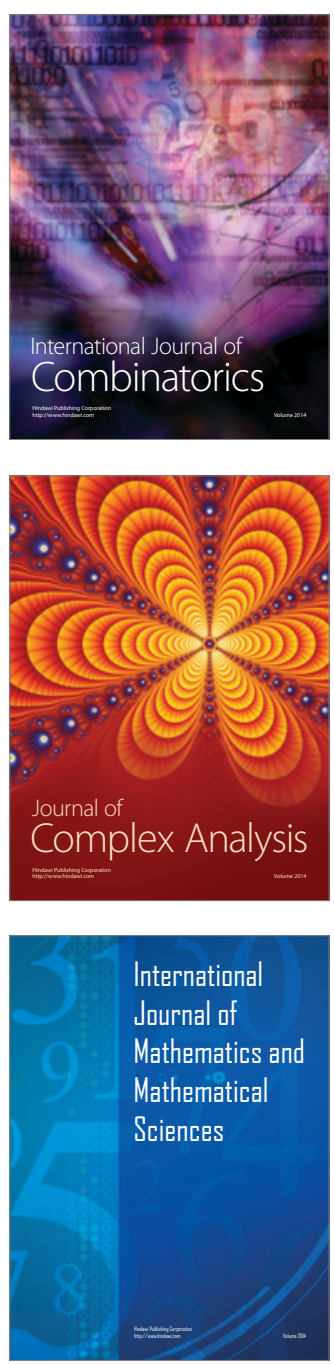
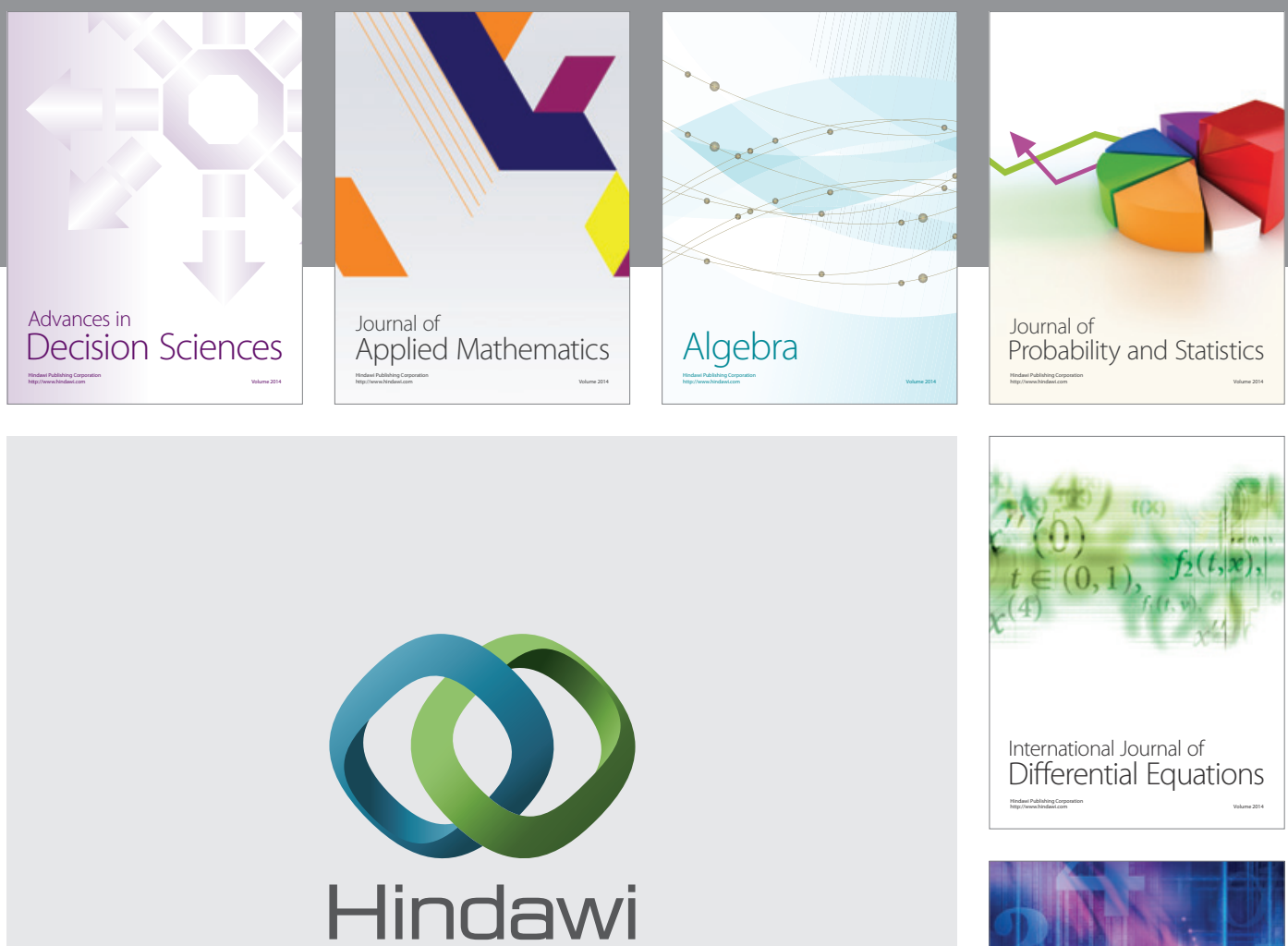

Submit your manuscripts at http://www.hindawi.com
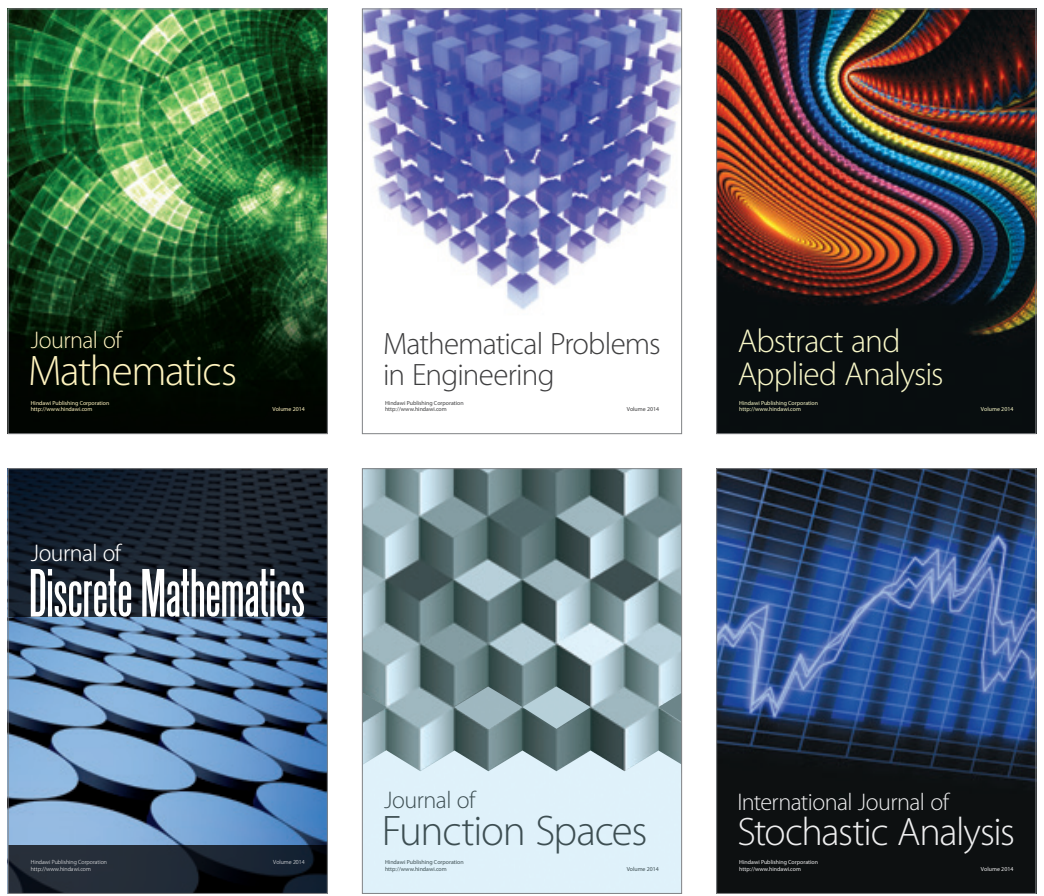

Journal of

Function Spaces

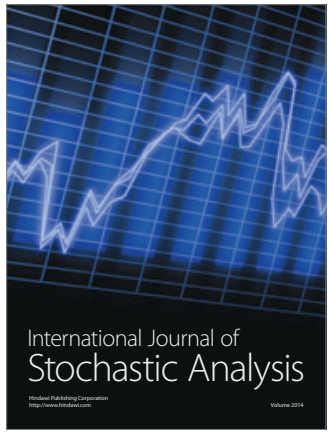

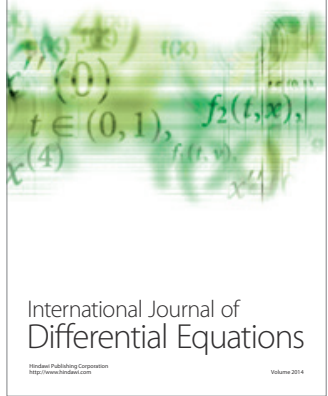
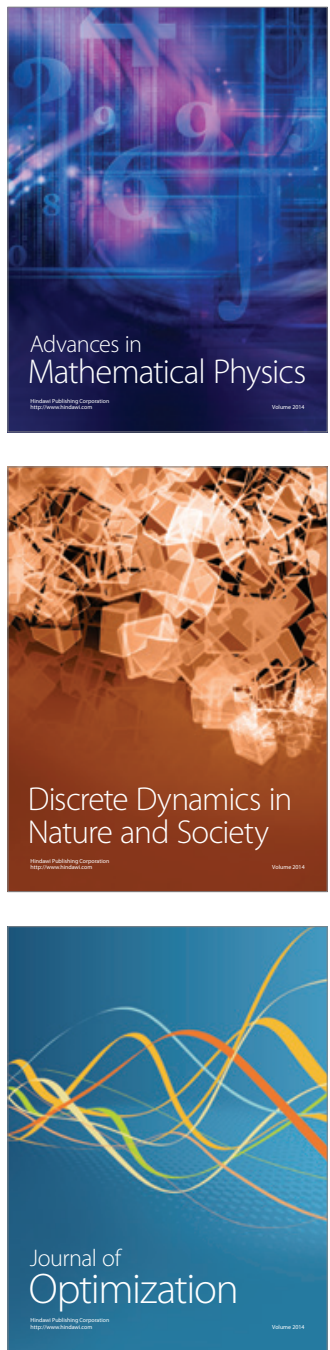